\title{
Supplementary Note
}

\section{Memberships of the International Genetics of Ankylosing Spondylitis (IGAS) Consortium}

The IGAS Consortium consists of the following people (excluding A.C. and M.A.B. already listed as authors):

Johanna Hadler ${ }^{1}$, Jenny P Pointon ${ }^{2}$, Philip C Robinson ${ }^{1}$, Tugce Karaderi ${ }^{2}$, Paul Leo ${ }^{1}$, Katie Cremin ${ }^{1}$, Karena Pryce ${ }^{1}$, Jessica Harris ${ }^{1}$, Seunghun Lee ${ }^{3}$, Kyung Bin Joo ${ }^{3}$, Seung-Cheol Shim ${ }^{4}$, Michael Weisman ${ }^{5}$, Michael Ward ${ }^{6}$, Xiaodong Zhou ${ }^{7}$, Henri-Jean Garchon ${ }^{8,9}$, Gilles Chiocchia ${ }^{8}$, Johannes Nossent $^{10,11}$, Benedicte A Lie ${ }^{12,13}, \varnothing$ ystein Førre ${ }^{14}$, Jaakko Tuomilehto ${ }^{15}$, Kari Laiho ${ }^{16}$, Lei Jiang ${ }^{17}$, Yu $\mathrm{Liu}^{17}{ }^{17}$, Xin Wu ${ }^{17}$, Linda A Bradbury ${ }^{1}$, Dirk Elewaut ${ }^{18}$, Ruben Burgos-Vargas ${ }^{19}$, Simon Stebbings ${ }^{20}$, Louise Appleton ${ }^{2}$, Claire Farrah ${ }^{2}$, Jonathan Lau ${ }^{2}$, Tony J Kenna ${ }^{1}$, Nigil Haroon ${ }^{21}$, Manuel A Ferreira ${ }^{22}$, Jian Yang $^{1}$, Juan Mulero ${ }^{23}$, Jose Luis Fernandez-Sueiro ${ }^{24}$, Miguel A Gonzalez-Gay ${ }^{25}$, Carlos Lopez-Larrea ${ }^{26}$, Panos Deloukas ${ }^{27}$, Peter Donnelly ${ }^{28}$, Australo-Anglo-American Spondyloarthritis Consortium (TASC), Groupe Française d'Etude Génétique des Spondylarthrites (GFEGS), Nord-Trøndelag health study (HUNT), Spondyloarthritis Research Consortium of Canada (SPARCC), Wellcome Trust Case-Control Consortium 2 (WTCCC2), Lianne Gensler ${ }^{29}$, Paul Bowness ${ }^{2}$, Karl Gafney ${ }^{30}$, Hill Gaston ${ }^{31}$, Dafna D Gladman $^{32,33,34}$, Proton Rahman ${ }^{35}$, Walter P Maksymowych ${ }^{36}$, Huji Xu ${ }^{17}$, J Bart A Crusius ${ }^{37}$, Irene E van der Horst-Bruinsma ${ }^{38}$, Chung-Tei Chou ${ }^{39,40}$, Raphael Valle-Oñate ${ }^{41}$, Consuelo Romero-Sánchez ${ }^{41}$, Inger Myrnes Hansen ${ }^{42}$, Fernando M Pimentel-Santos ${ }^{43}$, Robert D Inman ${ }^{21}$, Vibeke Videm ${ }^{44}, 45$, Javier Martin $^{46}$, Maxime Breban ${ }^{8,9}$, John D Reveille ${ }^{7}$, David M Evans ${ }^{47}$, Tae-Hwan Kim ${ }^{3}$, and Bryan Paul Wordsworth ${ }^{2}$

\footnotetext{
${ }^{1}$ University of Queensland Diamantina Institute, Translational Research Institute, Brisbane, Australia ${ }^{2}$ National Institute for Health Research (NIHR) Oxford Musculoskeletal Biomedical Research Unit, Nuffield Orthopaedic Centre, Headington, Oxford, United Kingdom

${ }^{3}$ Department of Rheumatology, Hanyang University Hospital for Rheumatic Diseases, Seoul, Republic of Korea

${ }^{4}$ Division of Rheumatology, Department of Medicine, Eulji University Hospital, Daejeon, Republic of Korea

${ }^{5}$ Department of Medicine/Rheumatology, Cedars-Sinai Medical Center, Los Angeles, California, USA ${ }^{6}$ National Institute of Arthritis and Musculoskeletal and Skin Diseases, National Institutes of Health, Bethesda, Maryland, USA

${ }^{7}$ Rheumatology and Clinical Immunogenetics, University of Texas Health Science Center at Houston, Houston, Texas, USA

${ }^{8}$ Institut Cochin, Université Paris-Descartes, CNRS (UMR 8104), INSERM U1016, France

${ }^{9}$ Ambroise Paré Hospital, Assistance Publique Hôpitaux de Paris, Versailles-Saint-Quentin en Yvelines University, Boulogne-Billancourt, France

${ }^{10}$ University Hospital North Norway, Tromso, Norway

${ }^{11}$ Division of Medicine, Royal Darwin Hospital, Darwin, Australia

${ }^{12}$ Department of Medical Genetics, University of Oslo and Oslo University Hospital, Oslo, Norway

${ }^{13}$ Department of Immunology, Oslo University Hospital, Oslo, Norway

${ }^{14}$ Department of Rheumatology, University Hospital Oslo, Oslo, Norway

${ }^{15}$ Department of Epidemiology and Health Promotion, National Public Health Institute, Helsinki, Finland

${ }^{16}$ Paijat-Hame Central Hospital, Lahti, Finland
} 
${ }^{17}$ Department of Rheumatology and Immunology, Shanghai Changzheng Hospital, Second Military Medical University, Shanghai 200003, China

${ }^{18}$ Department of Rheumatology, Gent University Hospital, Gent, Belgium

${ }^{19}$ Department of Rheumatology, Hospital General de México, Faculty of Medicine, Universidad Nacional Autónoma de México, México, México

${ }^{20}$ Department of Medicine, Dunedin School of Medicine, University of Otago, Dunedin, New Zealand

${ }^{21}$ Division of Rheumatology, Toronto Western Hospital, Toronto, Canada

${ }^{22}$ Queensland Institute of Medical Research, Royal Brisbane Hospital, Herston, Australia

${ }^{23}$ Rheumatology Department, Hospital Puerta de Hierro, Madrid, Spain

${ }^{24}$ Rheumatology Department, Complejo Hospitalario La Coruña, INIBIC, La Coruña, Spain

${ }^{25}$ Rheumatology Department, Hospital Marques de Valcecillas, IFIMAV, Santander, Spain

${ }^{26}$ Department of Immunology, Asturias Central University Hospital, Oviedo, Spain

${ }^{27}$ Wellcome Trust Sanger Institute, Cambridge, United Kingdom

${ }^{28}$ Wellcome Trust Centre for Human Genetics, University of Oxford, Oxford, United Kingdom

${ }^{29}$ University of California, San Francisco, 400 Parnassus Avenue, Box 0326, San Francisco, CA 941430326.

${ }^{30}$ Department of Rheumatology, Norfolk and Norwich University Hospital, Norwich, United Kingdom

${ }^{31}$ Department of Medicine, University of Cambridge, Addenbrookes Hospital, Cambridge, United Kingdom

${ }^{32}$ Division of Rheumatology, University of Toronto, Toronto, Canada

${ }^{33}$ Toronto Western Research Institute, Toronto, Canada

${ }^{34}$ Psoriatic Arthritis Program, University Health Network

${ }^{35}$ Memorial University of Newfoundland, Newfoundland, Canada

${ }^{36}$ Department of Medicine, University of Alberta, Canada

${ }^{37}$ Laboratory of Immunogenetics, Department of Medical Microbiology and Infection Control, VU University Medical Center, Amsterdam, The Netherlands

${ }^{38}$ Department of Rheumatology, VU University Medical Centre, Amsterdam, Netherlands

${ }^{39}$ Division of Allergy, Immunology, Rheumatology, Department of Medicine, Taipei Veterans General Hospital, Taipei, Taiwan

${ }^{40}$ School of Medicine, National Yang-Ming University, Taipei, Taiwan

${ }^{41}$ Spondyloarthropaty Group-Division of Rheumatology, Hospital Militar Central/ Universidad de La Sabana, Colombia

${ }^{42}$ Helgelandssykehuset, Mo i Rana, Norway

${ }^{43}$ Chronic Diseases Research Centre (CEDOC), Faculdade de Ciências Médicas, Universidade Nova de Lisboa, Portugal

${ }^{44}$ Department of Laboratory Medicine, Children's and Women's Health, Norwegian University of Science and Technology, Trondheim, Norway

${ }^{45}$ Department of Immunology and Transfusion Medicine, Tronheim University Hospital, Norway

${ }^{46}$ Instituto de Parasitología y Biomedicina López-Neyra, Consejo Superior de Investigaciones Científicas, Granada, Spain

${ }^{47}$ MRC Centre for Causal Analyses in Translational Epidemiology, School of Social and Community Medicine, Bristol, United Kingdom 